\title{
AVALIAÇÃO DAS PROPRIEDADES FÍSICO-QUÍMICAS E MECÂNICAS DE FILMES DE FÉCULA DE MANDIOCA INCORPORADO COM CAFEÍNA IRRADIADA.
}

Evaluation of physical-chemical and mechanical properties of cassava starch films incorporated with irradiated caffeine

Evaluación de las propriedades físico-químicas y mecânicas de películas de almidón de mandioca incorporado con cafeína irradiada

\section{Maria Carolina Santos Silva*1, Pablo Kashisol Duarte de Lima', Miriam Fontes Araújo Silveira², Valter Arthur $^{3}$, Adriana Régia Marques de Souza ${ }^{2}$}

${ }^{1}$ Discente do curso de Engenharia de Alimentos, Universidade Federal de Goiás, Goiânia, Brasil.

${ }^{2}$ Docente do curso de Graduação em Engenharia de Alimentos, Universidade Federal de Goiás, Goiânia, Brasil.

${ }^{3}$ Centro de Energia Nuclear na Agricultura, Universidade de São Paulo, Piracicaba, Brasil

*Correspondência: Escola de Agronomia, Rodovia Goiânia-Nova Veneza, Km 0, s/n - Campus Samambaia, Goiânia, Goiás, Brasil.CEP: 74690900.e-mail drilavras@yahoo.com.br

Artigo recebido em 29/04/2019 aprovado em 03/05/2019 publicado em 16/06/2019.

\section{RESUMO}

As embalagens biodegradáveis são provenientes de fontes renováveis, como milho, celulose e mandioca, sendo que essas matérias primas, quando associadas a outros materiais para produção de filmes biodegradáveis, podem ocasionar mudanças nas propriedades mecânicas e de barreira do produto final. A produção de embalagens ativas pode contribuir na conservação de alimentos, aumentando sua vida útil e preservando suas características físicas, químicas e sensoriais, um componente utilizado em embalagens ativas é a cafeína, que possui atividade antimicrobiana. O objetivo do presente trabalho foi produzir filmes de fécula de mandioca incorporados com cafeína irradiada e analisar possíveis alterações quanto as propriedades físico-químicas e mecânicas. Verificou-se que a espessura, a solubilidade em água, permeabilidade ao vapor de água e o ensaio de tração dos filmes não apresentaram diferença significativa entre os tratamentos $(p>0,05)$. Porém houve alteração de cor e de resistência a perfuração dos filmes com a incorporação de cafeína irradiada, onde os filmes apresentavam-se mais frágeis e com coloração mais escura a medida que as doses de radiação aplicadas foram aumentando. A incorporação de cafeína irradiada permitiu a obtenção de um filme de boa qualidade mecânica, porém as doses estudadas não apresentou efeito nas propriedades dos mesmos.

Palavras-chave: biodegradável, radiação gama, amido.

\section{ABSTRACT}

Biodegradable packaging comes from renewable sources such as corn, cellulose and cassava. They are raw materials that when associated to other materials, for the production of biodegradable films, may present changes in mechanical and barrier properties of final product. The production of active packaging can contribute to the preservation of food, increasing its shelf life and preserving its physical, chemical and sensorial characteristics, a component used in active packaging is caffeine, which has antimicrobial activity. The objective of the present work was to produce cassava starch films incorporated with irradiated caffeine and to analyze possible changes in physicochemical and mechanical properties. It was verified that the thickness, water solubility, water vapor permeability and the tensile strength of the films did not show significant difference between the treatments ( $p>0.05)$. However, there was a change in color and perforation resistance of the films with the incorporation of irradiated caffeine, where the films were more fragile and darker colored as the applied radiation doses were increased. The 
incorporation of irradiated caffeine allowed to obtain a film of good mechanical quality, but the doses studied did not show effect on the properties of the same ones.

Keywords: biodegradable, gamma radiation, starch.

\section{RESUMEN}

Los envases biodegradables proceden de fuentes renovables, como el maíz, la celulosa y la mandioca, siendo que estas materias primas, cuando se asocian a otros materiales para la producción de películas biodegradables, pueden ocasionar cambios en las propiedades mecánicas y de barrera del producto final. La producción de envases activos puede contribuir a la conservación de alimentos, aumentando su vida útil y preservando sus características físicas, químicas y sensoriales, un componente utilizado en envases activos es la cafeína, que posee actividad antimicrobiana. El objetivo del presente trabajo fue producir películas de fécula de mandioca incorporadas con cafeína irradiada y analizar posibles alteraciones en cuanto a las propiedades físico-químicas y mecánicas. Se verificó que el espesor, la solubilidad en agua, la permeabilidad al vapor de agua y el ensayo de tracción de las películas no mostraron diferencia significativa entre los tratamientos ( $p>0,05)$. Pero hubo alteración de color y de resistencia a la perforación de las películas con la incorporación de cafeína irradiada, donde las películas se presentaban más frágiles y con coloración más oscura a medida que las dosis de radiación aplicadas fueron aumentando. La incorporación de cafeína irradiada permitió la obtención de una película de buena calidad mecánica, pero las dosis estudiadas no tuvieron efecto en las propiedades de los mismos.

Descriptores: biodegradable, radiación gamma, almidón.

\section{INTRODUÇÃO}

Os filmes na área de alimentos funcionam como barreira para transferência de gases e umidade, além de auxiliar na diminuição da degradação dos produtos alimentícios, causados por fatores ambientais. A maioria dos filmes utilizados são elaborados com materiais plásticos, polímero de difícil degradação e proveniente de fonte não renovável (COURTNEY; MUNGUÍA, 2012). Com o avanço do crescimento populacional, se tornou uma preocupação atender toda demanda alimentar considerando os meios de conservação de alimentos com filmes de plástico, havendo a necessidade de desenvolver alternativas, como as embalagens biodegradáveis advindo de fontes renováveis, abundantes e econômicas que tenham a capacidade de interagir com o alimento afim de prolongar sua vida de prateleira com qualidade, garantindo assim a segurança alimentar de seus consumidores.

Embalagens biodegradáveis, também conhecidas como biofilmes, são desenvolvidas por polímeros, de fontes naturais, como milho, celulose, mandioca, etc. Nos quais a degradação ocorre por microrganismos em semanas ou meses sob condições favoráveis de biodegradação (BRITO, 2011). Segundo Luvielmo e Lamas (2012), a mandioca é uma matériaprima de grande potencial na elaboração de filmes e revestimentos comestíveis por ser de baixo custo e por formar películas resistentes e transparentes que proporcionam eficiente barreira a gases. Nesses biofilmes ainda podem ser incorporados aditivos alimentares como antimicrobianos, enzimas, antioxidantes e aromatizantes, que visam melhorar a qualidade, com aumento da vida de prateleira dos alimentos e suas características sensoriais.

A grande exigência do consumidor por alimentos naturais e saudáveis faz com que as indústrias de alimentos se modifiquem, investindo em pesquisas e tecnologias avançadas para garantir ao consumidor produtos similar ao in natura. A produção de embalagens ativas pode contribuir na conservação de alimentos, aumentando sua vida útil e preservando suas características físicas, químicas e sensoriais. A cafeína é uma substancia inodora, solúvel em água, pouco volátil, termicamente estável e farmacologicamente ativo (POSSER, 2016). Está 
presente em café, chá verde, mate, dentre outros, além de possuir propriedades distintas, como efeito estimulante, atividade antimicrobiana e, principalmente, atividade antioxidante e inibitória de atividade enzimática (MARIA; MOREIRA, 2007).

A irradiação de alimentos consiste no uso de radiação ionizante aplicada à alimentos, a fim de conservação ou esterilização comercial, sendo utilizados fontes de ${ }^{60} \mathrm{Co}$ e ${ }^{137} \mathrm{Cs}$, que são consideradas de uso comercial (SILVA; ROZA, 2010). Silva (2012) avalia o efeito da radiação gama $60 \mathrm{Co}$, a doses de 5 e 10 kGy, em sementes de café e relata que não houve diferença significativa na composição química do composto cafeína. Fanaro (2013) argumenta também que a cafeína, de chá verde da planta Camellia sinensis, se mantém estável frente à radiação em diferentes doses, mas o chá preto da planta Camellia sinensis, a dose de $10 \mathrm{kGy}$, tem diferença considerável na composição química deste composto.

Tendo em vista a carência no mercado de biofilmes capazes de proporcionar uma maior viabilidade dos alimentos, o objetivo desse presente trabalho foi produzir filmes de fécula de mandioca incorporados com cafeína submetida a diferentes doses de radiação gama e avaliar as alterações físicoquímicas e propriedades mecânicas.

\section{MATERIAIS E MÉTODOS}

Os experimentos foram conduzidos nos laboratórios do Setor de Engenharia de Alimentos, da Escola de Agronomia, da Universidade Federal de Goiás.

\section{1 - Irradiações da Cafeína}

A cafeína foi irradiada nas doses de 0 (controle), 2, 4, 8 e 16 kGy em um irradiador Gamacell com fonte de 60Co, no Centro de Energia Nuclear na
Agricultura (CENA-USP) na cidade de Piracicaba SP.

\section{2 - Elaboração dos filmes}

Os filmes foram produzidos pelo método casting (FERNANDES, 2014) com algumas modificações. As soluções filmogênicas foram preparadas contendo $10 \%$ de fécula de mandioca, $4 \%$ de glicerol, $85 \%$ de água e $1 \%$ de cafeína, variando este componente de acordo com os tratamentos.

A produção do filme consistiu primeiramente na solubilização da cafeína em água previamente aquecida. Em seguida foram adicionados o amido e glicerol, sendo a solução aquecida em chapa, atingindo aproximadamente, $85^{\circ} \mathrm{C}$, onde ocorreu a gelificação total da fécula de mandioca. Posteriormente $50 \mathrm{~g}$ das soluções filmogênicas preparadas foram dispersas em placas de vidro, encapadas com plástico adesivo. As placas então foram colocadas em estufa com circulação e renovação de ar (Marconi - MA O35, Piracicaba, Brasil), à temperatura de $45^{\circ} \mathrm{C}$ por 5 horas. Os filmes foram condicionados em dessecador até atingir temperatura ambiente, sendo retirados e identificados para análises subsequentes.

\section{3 - Analises de caracterização do filme}

\subsection{1 - Espessura}

As medidas de espessura dos filmes foram efetuadas utilizando um micrômetro com sensibilidade de 0,001 mm (Insize, 32110-25, China), sendo obtida através da média dos valores de 10 pontos aleatórios em diferentes segmentos do filme.

\subsection{2-Cor}

A cor foi determinada em aparelho ColorQUEST XE (Hunterlab, Estados Unidos, serie: CQX4181), operando no padrão $L^{*} a^{*} b^{*}$ CIE (Commission Internacional de L' Eclairage). As coordenadas medidas, em refletância especular incluída, foram: $\mathrm{L}^{*}$ (luminosidade) a* (tonalidades de 
verde a vermelho) e b* (tonalidades de azul a amarelo). Com os dados coletados calcularam-se os valores de croma ou $\mathrm{C}^{*}$ e do Hab ou ângulo de tonalidade. Pelas equações (1) e (2):

$$
\begin{aligned}
& \mathrm{C}^{*}=\left(\left(\mathrm{a}^{*}\right)^{2}+\left(\mathrm{b}^{*}\right)^{2}\right)^{1 / 2}(\text { Eq. 1) } \\
& \mathrm{H}_{\mathrm{ab}}=\tan ^{-1}\left(\mathrm{~b} * / \mathrm{a}^{*}\right) \quad(\text { Eq. } 2)
\end{aligned}
$$

\subsection{3 - Solubilidade em água}

Porções de filmes foram secos em estufa (Tecnal - TE-395, Brasil) a $105^{\circ} \mathrm{C}$ por 24 horas para determinação da matéria seca inicial dos filmes. Após a primeira pesagem as amostras foram colocadas em tubo falcon, com $50 \mathrm{~mL}$ de água destilada e agitadas lentamente por 24 horas em homogeneizador de soluções (Phoenix - AP22, Araraquara, Brasil). As amostras foram removidas e secas em estufa (Tecnal TE-395, Piracanjuba, Brasil) a $105^{\circ} \mathrm{C}$ por 24 horas para determinar a massa de matéria seca que não dissolveu em água. O percentual de solubilidade dos filmes foi determinado como porcentagem de matéria seca solubilizada (GONTARD et al., 1994; FERNANDES, 2014).

\subsection{4 - Permeabilidade a Vapor de Água (PVA)}

A permeabilidade ao vapor de água (PVA) foi determinada de acordo com o método gravimétrico E96/E96 da ASTM (2010) a $25^{\circ} \mathrm{C}$, com modificações. As amostras dos filmes foram cortadas na forma de discos e seladas em cápsulas de permeação feitas de polipropileno, contendo cloreto de cálcio anidro seco em estufa à $200^{\circ} \mathrm{C}$. As cápsulas de permeação foram acondicionadas em dessecador (Tecnal, TE-3950, Brasil) contendo solução salina saturada de cloreto de sódio (umidade relativa de $75 \%$ ).

O cloreto de cálcio anidro é um dessecante, com alta afinidade ao vapor de água, o que permite quantificar o ganho de massa, ou seja, a quantidade de vapor de água que permeia pelo material a ser testado (RIBEIRO, 2014). O ganho de massa no sistema foi verificado periodicamente em intervalos de 30 minutos durante as primeiras $2 \mathrm{~h}$, a cada $2 \mathrm{~h}$ durante as $8 \mathrm{~h}$ seguintes e, finalmente, após 24h. Mudanças no ganho de massa (g) foi registrado e construído um gráfico em função do tempo (h) e, da reta obtida por regressão linear, foi determinado o coeficiente angular e calculada a taxa de permeabilidade ao vapor de água (TVA) (FERNANDES, 2014), de acordo com a equação1.

$$
T V A=\frac{\mathrm{g} / \mathrm{t}}{A} \quad(\mathrm{Eq} .3)
$$

Onde:

TVA: taxa de permeabilidade ao vapor de água (g/h.m2)

$\mathrm{g} / \mathrm{t}$ : coeficiente angular da reta $(\mathrm{g} / \mathrm{h})$

A: área de permeação (m2)

Em seguida, a permeabilidade ao vapor de água (PVA) foi calculada utilizando-se a equação 2 (FERNANDES, 2014):

$$
P V A=\frac{\mathrm{TVA} * \mathrm{X}}{\Delta \mathrm{P}}(\text { Eq. } 4)
$$

Onde:

PVA: permeabilidade ao vapor de água $\left(\mathrm{g}^{*} \mathrm{~mm} / \mathrm{h}\right.$. $\mathrm{m} 2 \mathrm{kPa})$

$\mathrm{x}$ : espessura média do filme (mm)

$\Delta \mathrm{P}$ : diferença de pressão de vapor do ambiente $(\mathrm{kPa})$ contendo cloreto de cálcio anidro ( $2 \%$ de UR) e solução saturada de $\mathrm{NaCl}$ (75\% de UR).

\subsection{5 - Propriedades Mecânicas}

\subsubsection{1 - Tração}

As propriedades mecânicas dos filmes foram avaliadas pelo ensaios de perfuração em texturômetro (Stable Micro System, TA.XT.plus Texture Analyser, Surrey, Inglaterra) e o ensaio de tração em máquina universal de ensaios (Instron, modelo 3367, Grove City).

Para o ensaio de tração foram preparadas tiras de amostras, de cada tratamento, com largura de 15 $\mathrm{mm}$, comprimento de $120 \mathrm{~mm}$. As amostras foram 
acondicionadas em envelopes de papel, em dessecador e temperatura ambiente por $48 \mathrm{~h}$. O equipamento foi ajustado com espaço entre ganchos de $100 \mathrm{~mm}$. As amostras foram tracionadas com velocidade de $0,21 \mathrm{~mm} / \mathrm{s}$, utilizando-se no equipamento uma célula de carga com capacidade de 500 N. Analisaram-se a tensão máxima, tensão na ruptura, o alongamento ou extensão máxima e o módulo de elasticidade ou módulo de Young (CARVALHO, 2017).

\subsubsection{2 - Perfuração}

Para o ensaio de perfuração as amostras foram cortadas em formato circular, fixadas no probe e perfuradas com uma sonda deslocando-se a $0,21 \mathrm{~mm} / \mathrm{s}$ até perfuração dos filmes. Analisaram-se a força máxima na ruptura do filme.

\section{4 - Análise Estatística}

Os dados foram submetidos à Análise de Variância (ANOVA) e as diferenças entre os tratamentos avaliados pelo teste de médias Tukey à 5\% de probabilidade, com auxílio do programa STATISTICA versão 10 (StatSoft, Inc.,2011). O delineamento experimental foi em blocos casualizados e as análises foram feitas com três repetições lidas em triplicatas.

\section{RESULTADOS E DISCUSSÃO}

\section{1 - Caracterização dos filmes}

Os valores médios e os desvios-padrão da espessura, solubilidade em água e permeabilidade ao vapor de água (PVA) dos filmes de fécula de mandioca incorporado com cafeína irradiada, em diferentes doses, (Tabela 1) não apresentaram diferença significativa $(\mathrm{p}>0,05)$ entre os tratamentos.

Não houve variação na espessura dos filmes produzidos independente do tratamento, sendo que uma maior uniformidade implica em uma melhor confiança nos resultados das análises subsequentes, pois a espessura pode influenciar nas propriedades mecânicas e de barreira do filme, comprometendo o desempenho da embalagem (SILVA, 2004). Versino e Garcia (2014) produziram filmes de amido e glicerol e obtiveram espessura de $0,067 \mathrm{~mm}$. Henrique, et al (2008), elaboraram biofilmes com concentração 3 e $5 \%$ de amido de mandioca modificado e obtiveram filmes com espessura de 0,060 a 0,120mm, valores abaixo do observado nos filmes de fécula de mandioca incorporado com cafeína. Contudo Friedrich (2017) produziu filmes de amido de mandioca e observou valores de $0,154 \mathrm{~mm}$ para o filme com amido e glicerol e $0,373 \mathrm{~mm}$ para filmes com adição de agente antimicrobiano, tornando os filmes mais espessos e consequentemente mais resistentes.

Verificou-se ainda que a solubilidade em água e a permeabilidade ao vapor de água demonstraram que a cafeína submetida a irradiação não influenciou nas propriedades de barreira e de interação hidrofóbica e hidrofílica da água com o filme de fécula de

Tabela 1. Espessura, solubilidade em água e permeabilidade ao vapor de água dos filmes de

fécula de mandioca incorporado com cafeína irradiada.

\begin{tabular}{cccc}
\hline TRATAMENTO $(\mathrm{kGy})$ & ESPESSURA $(\mathrm{mm})$ & SOLUBILIDADE $(\%)$ & PVA $\left(\mathrm{g} \cdot \mathrm{mm} / \mathrm{h} \cdot \mathrm{m}^{2} \cdot \mathrm{kPa}\right)$ \\
\hline 0 & $0,1960 \pm 0,0082^{\mathrm{a}}$ & $29,8633 \pm 3,8040^{\mathrm{a}}$ & $0,4060 \pm 0,0653^{\mathrm{a}}$ \\
2 & $0,2034 \pm 0,0090^{\mathrm{a}}$ & $33,0733 \pm 2,7919^{\mathrm{a}}$ & $0,3934 \pm 0,0781^{\mathrm{a}}$ \\
4 & $0,1925 \pm 0,0086^{\mathrm{a}}$ & $40,5767 \pm 4,3694^{\mathrm{a}}$ & $0,4309 \pm 0,0430^{\mathrm{a}}$ \\
8 & $0,1783 \pm 0,0067^{\mathrm{a}}$ & $31,0900 \pm 1,8355^{\mathrm{a}}$ & $0,4638 \pm 0,1073^{\mathrm{a}}$ \\
16 & $0,1919 \pm 0,0089^{\mathrm{a}}$ & $34.3000 \pm 2,5720^{\mathrm{a}}$ & $0,4319 \pm 0,0869^{\mathrm{a}}$
\end{tabular}

*Médias seguidas de letras iguais nas colunas não diferem estatisticamente entre si $(p>0,05)$. 
mandioca. Batista (2014) encontrou para filmes de amido de mandioca uma solubilidade de 46,85\%. Enquanto Vicentini (2003), relatou uma baixa solubilidade de filmes de fécula de mandioca, com valor encontrado de 4\%, com espessura acima de 0,040mm, porém atribuiu essa baixa ao não uso de plastificante na composição do filme.

Assim como os demais parâmetros analisados, a permeabilidade ao vapor de água não apresentou diferença entre os tratamentos. Farias (2016) elaborou filmes de amido de mandioca incorporado com polpa de acerola e óleo essencial de cravo e encontrou valores entre 0,36 e $0,39 \mathrm{~g} \cdot \mathrm{mh} \cdot \mathrm{m}^{2} \cdot \mathrm{kPa}$, mantendo a concentração de $4 \%$ de amido e variando os outros componentes, porém não evidenciou diferença significativa. Silva e Cortez-Verga (2014), elaboraram luminosidade, ou seja, indicou que os filmes estão mais claros. O croma indicou uma diminuição na intensidade de cor para os filmes com cafeína irradiada.

Foi evidente a diferença de cor em função da incorporação de cafeína irradiada no biofilme de fécula de mandioca. O controle (0kGy) apresentou maior luminosidade e menor parâmetro de croma, indicando filme mais escuro e de maior intensidade de cor, em comparação com os filmes incorporado com cafeína irradiada. A cafeína é um composto orgânico nitrogenado, sendo um metabolito secundário das plantas. Segundo Inamura (2008) a radiação ionizante pode produzir radicais livres, como hidroxila, que quando reagem com um alimento ou substancia, são capazes de produzir compostos indesejáveis,

Tabela 2. Coloração dos filmes de fécula de mandioca incorporado com cafeína irradiada

\begin{tabular}{cccccc}
\hline TRATAMENTO & $\mathrm{L}$ & $\mathrm{a}^{*}$ & $\mathrm{~b}^{*}$ & $\mathrm{C}^{*}$ & $\mathrm{~h}_{\mathrm{ab}}$ \\
\hline 0 & $46,78 \pm 2,68^{\mathrm{a}}$ & $-0,86 \pm 0,02^{\mathrm{a}}$ & $-5,19 \pm 0,14^{\mathrm{a}}$ & $5,26 \pm 0,14^{\mathrm{a}}$ & $1,405 \pm 0,003^{\mathrm{a}}$ \\
2 & $58,68 \pm 0,45^{\mathrm{b}}$ & $-0,75 \pm 0,05^{\mathrm{b}}$ & $-4,27 \pm 0,24^{\mathrm{b}}$ & $4,34 \pm 0,24^{\mathrm{b}}$ & $1,399 \pm 0,002^{\mathrm{b}}$ \\
4 & $59,65 \pm 2,33^{\mathrm{c}}$ & $-0,77 \pm 0,02^{\mathrm{c}}$ & $-4,25 \pm 0,05^{\mathrm{c}}$ & $4,32 \pm 0,05^{\mathrm{c}}$ & $1,390 \pm 0,006^{\mathrm{c}}$ \\
8 & $56,41 \pm 1,03^{\mathrm{d}}$ & $-0,78 \pm 0,03^{\mathrm{d}}$ & $-4,80 \pm 0,12^{\mathrm{d}}$ & $4,86 \pm 0,12^{\mathrm{d}}$ & $1,411 \pm 0,003^{\mathrm{d}}$ \\
16 & $53,86 \pm 1,70^{\mathrm{a}}$ & $-0,76 \pm 0,03^{\mathrm{bc}}$ & $-4,78 \pm 0,18^{\mathrm{e}}$ & $4,84 \pm 0,18^{\mathrm{e}}$ & $1,412 \pm 0,003^{\mathrm{d}}$
\end{tabular}

*Médias seguidas de letras iguais nas colunas não diferem estatisticamente entre $s i(p>0,05)$.

filmes de fécula de mandioca, com concentração de $3 \%$ variando o plastificante e obtiveram valores entre 0,38 a 2,29 g.mm $/ \mathrm{m}^{2}$.dia.kPa e perceberam que a adição do glicerol favoreceu no aumento do permeabilidade ao vapor de água.

\section{2-Cor}

Os parâmetros de cor avaliados para os filmes de fécula de mandioca incorporada com cafeína irradiada nas doses de 0, 2, 6, 8 e $16 \mathrm{kGy}$ (Tabela 2) demostrou que houve diferença significativa $(p>0,05)$ para todos os valores medidos de $\mathrm{L}, \mathrm{a}^{*}, \mathrm{~b}^{*}$ e calculados de $C^{*}$ e Hab.

Observou-se que os filmes com cafeína irradiada tenderam a apresentar maiores valores para comprometendo funcionalidades, destruindo nutrientes, dentre outros.

\section{3 - Propriedades Mecânicas}

\subsection{1 - Tração:}

Os parâmetros analisados do teste de tração (Tabela 3), demostrou que a cafeína irradiada a diferentes doses não influenciou na resistência a ruptura do filme. E também não se evidenciou uma mudança de elongamento desse filme.

No modulo de elasticidade foi verificado uma diferença estatística entre os tratamentos de 2 e 8 kGy, 
Tabela 3. Valores das análises de tração dos filmes de fécula de mandioca incorporado com cafeína irradiada.

\begin{tabular}{cccc}
\hline $\begin{array}{c}\text { TRATAMENTO } \\
(\mathrm{kGy})\end{array}$ & $\begin{array}{c}\text { Tensão na } \\
\text { Ruptura (MPa) }\end{array}$ & $\begin{array}{c}\text { Alongamento } \\
(\%)\end{array}$ & $\begin{array}{c}\text { Módulo de } \\
\text { Elasticidade (MPa) }\end{array}$ \\
\hline 0 & $1,222 \pm 0,241^{\mathrm{a}}$ & $4,780 \pm 0,547^{\mathrm{a}}$ & $172,019 \pm 26,589^{\mathrm{ab}}$ \\
2 & $1,479 \pm 0,350^{\mathrm{a}}$ & $4,351 \pm 0,439^{\mathrm{a}}$ & $214,244 \pm 41,600^{\mathrm{a}}$ \\
4 & $1,140 \pm 0,244^{\mathrm{a}}$ & $3,792 \pm 0,398^{\mathrm{a}}$ & $164,780 \pm 26,588^{\mathrm{ab}}$ \\
8 & $0,785 \pm 0,261^{\mathrm{a}}$ & $4,805 \pm 0,418^{\mathrm{a}}$ & $146,630 \pm 29,812^{\mathrm{b}}$ \\
16 & $1,160 \pm 0,298^{\mathrm{a}}$ & $4,559 \pm 0,446^{\mathrm{a}}$ & $191,312 \pm 31,796^{\mathrm{ab}}$
\end{tabular}

*Médias seguidas de letras iguais nas colunas não diferem estatisticamente entre $s i(p>0,05)$.

porém essa diferença não foi evidenciada em comparação ao filme controle $(0 \mathrm{kGy})$, com cafeína não irradiada, de fécula de mandioca.

Foi observado que os filmes de fécula de mandioca incorporados com cafeína irradiada assumiram valores entre 3,792 a 4,805\% para o alongamento e 0,787 a 1,479MPa de tensão de ruptura. Valores abaixo do observado por Sueiro (2016) que resulta em um alongamento de $33 \%$ e uma resistência máxima a tração de 4,82MPa para filmes de amido de mandioca à $3 \%$ com a adição de $0,20 \mathrm{~g}$ de glicerol/g de

\subsection{2 -Perfuração:}

$\mathrm{Na}$ análise de perfuração dos filmes com cafeína irradiada foi observado que houve diferença significativa $(\mathrm{p}<0,05)$. Sendo a dose aplicada de 16 kGy diferente significativamente do controle ( $0 \mathrm{kGy})$ e de 2 kGy de cafeína irradiada. $\mathrm{O}$ aumento nas doses de radiação promoveu diminuição nos valores de força máxima aplicada na ruptura (Tabela 4).

Sendo observado que os filmes de fécula de mandioca se apresentaram mais frágeis com a utilização de cafeína, submetida a maiores doses de

Tabela 4. Valores da analise de perfuração dos filmes

\begin{tabular}{cc}
\hline TRATAMENTO (kGy) & Força Máxima da Ruptura (N) \\
\hline 0 & $5,7698 \pm 0,5542^{\mathrm{a}}$ \\
2 & $5,3534 \pm 0,6551^{\mathrm{a}}$ \\
4 & $4,6209 \pm 0,3748^{\mathrm{ab}}$ \\
8 & $4,7906 \pm 0,4584^{\mathrm{ab}}$ \\
16 & $3,5749 \pm 0,3099^{\mathrm{b}}$
\end{tabular}

*Médias seguidas de letras iguais nas colunas não diferem estatisticamente entre si $(p>0,05)$.

biopolímero. Enquanto Santos (2014) encontra 81,2\% de alongamento e 3,7Mpa de tensão na ruptura para filmes produzidos com a mesma concentração de amido e glicerol. Assim o tipo de amido, a quantidade utilizada de plastificante e de aditivo, como a cafeína, na formulação, podem ter influenciados nas propriedades de tração do filme, porém não foi possível verificar diferença entre os tratamentos com cafeína irradiada. radiação, devido a menor força requerida para ruptura.

Segundo Vicentini (2003), o filme deve apresentar certa resistência a ruptura e a abrasão, também uma certa flexibilidade, que permita a deformação do filme sem a sua ruptura, sendo características importantes para embalagens do tipo biofilme. De acordo com Junior (2009) a resistência do filme à perfuração está relacionada com o estado de compactação da rede polimérica. Vicentini (2003) relata que a força na ruptura é influenciada também pela espessura do filme, e observa que filmes de fécula de mandioca são mais resistentes que outros tipos de 
filmes como os filmes de amido de cará e de proteínas miofibrilares. Farias (2016) sugere ainda que a presença de aditivos e plastificantes no filme pode alterar as interações polímero-polímero, trazendo a uma diminuição, ou não, da mobilidade das cadeias poliméricas e consequentemente tornando os filmes menos elásticos e mais resistentes ou mais elásticos e menos resistentes, Logo, como não houve alteração da concentração de glicerol e de amido e não houve diferença estatística quanto a espessura, podemos inferir que quanto maior for a dose de radiação aplicada na cafeína, mais frágil se torna o filme de fécula de mandioca produzido, pela aplicação de uma força $(\mathrm{N})$ e consequentemente os tornando menos elásticos.

\section{CONCLUSÃO}

A incorporação de cafeína irradiada permitiu a obtenção de um filme de boa qualidade mecânica, porém as doses estudadas não apresentou efeito nas propriedades dos mesmos.

Todos os autores declararam não haver qualquer potencial conflito de interesses referente a este artigo.

\section{REFERÊNCIAS}

AMERICAN SOCIETY FOR TESTING AND MATERIALS-ASTM. Standard Test Methods for Water Vapor Transmission of Materials - ASTM E96/E96M-10. Filadelfia, PA (USA): American Society for Testing and Materials. 2010.

BATISTA, M. P., Fernandes, A.S., Ascheri, D.P.R., Sousa, Y.S. Filmes biodegradáveis de amido de lírio-do-brejo, milho, batata e mandioca: comparação da solubilidade, permeabilidade $e$ testes mecânicos. In: $54^{\circ}$ Congresso Brasileiro de Químico. 2014. Disponível em: <http://www.abq.org.br/cbq/2014/trabalhos/9/513719197.html >. Acesso em: 20 de jul de 2018.

BRITO, G. F., AGRAWAL, P., ARAÚJO, E.M., MÉLO,J.J.A.,Biopolímero, Polímeros Biodegradáveis e Polímeros Verdes. Revista Eletrônica de Materiais e Processos. v.6, n.2, p.127-139, 2011.

CARVALHO, D. M., TAKEUCHI, K. P., GERALDINE, R. M., MOURA, C. J., SILVEIRA, M. F. A. Filme ativo de acetato de celulose incorporado com nanosuspensão de curcumina. Polímeros, v.27. p.70-76, 2017.

DOMÍNAGUEZ-COURTNEY, M. F; JIMÉNEZMUNGUÍA, M. T. Películas comestibles formulados con polisacáridos: propiedades y aplicaciones. Temas Selectos de Ingenería de Alimentos. v.6, n.2, p.110121. 2012.

FANARO. G. B. Efeito da radiação ionizante em chás da planta Camellias sinensis irradiados com diferentes atividades de água. Tese (Doutor em Ciências), Instituto de Pesquisas Energéticas e Nucleares. São Paulo, 2013.

FARIAS, M. G. Elaboração e caracterização de filmes de amido e polpa de acerola por casting, extrusão termoplástica e termoprensagem. Tese (Doutor em Ciências) - Universidade Federal Rural do Rio de Janeiro. Seropédica, 2016.

FERNANDES. A. P. Avaliação e caracterização das propriedades filmogênicas de proteínas de soro de leite submetidos à irradiação e modificação de pH. Dissertação (Mestre em Ciência e Tecnologia de Alimentos), Escola de Agronomia, Universidade Federal de Goiás. Goiânia, 2014.

FERREIRA, A. H. Efeito da adição de surfactantes e do ajuste de $\mathbf{p H}$ sobre filmes a base de gelatina, triactina, ácidos graxos e ceras de carnaúba e de cana-de-açúcar. Dissertação (Mestre em Alimentos e Nutrição), Faculdade de Engenharia de Alimentos Universidade Estadual de Campinas. Campinas, 2006.

FRIEDRICH, J. C. C. Biofilmes a base de amido, gelatina e extrato de Tetradenia Riparia na conservação de Morango. Dissertação (Mestre em Ciências Ambientais). Universidade Estadual do Oeste do Paraná. Toledo, 2017.

GORTAND, N., DUCHEZ, C.; CUQ, J-L.; GUILBERT, S. Edible composite films of wheat gluten and lipids: water vapour permeability and other physical properties. Internacional Journal of Food Science and Technology, v.29, .39-50. 1994.

HENRIQUE, C. M. CEREDA, M. P. SARMENTO, S. B. S. Características físicas de filmes biodegradáveis produzidos a partir de amidos modificados de mandioca. Ciência e Tecnologia de Alimentos. Campinas, v.28, n.1, p.231-2410. 2008 
INAMURA, P. Y. Efeito da radiação dama sobre a viscosidade de soluções de gelatina e pectina, utilizadas na indústria de alimentos. Dissertação (Mestre em Ciência), Instituto de Pesquisas Energéticas e Nucleares. São Paulo, 2008.

JUNIOR, M. D. M. Caracterização de biofilmes obtidos a partir de amido de ervilha (Pisum Sativum) associado à goma xantana e glicerol. Dissertação (Mestrado em Ciências). Escola Superior de Agricultura Luiz de Queiroz - Universidade de São Paulo. Piracicaba, 2009.

LUVIELMO, M. M. LAMAS, S. V. Revestimento comestível em frutas. Estudos Tecnológicos em Engenharia, v.8, n.1, p.8-15. 2012.

MARIA, C.A.B.; MOREIRA, R.F.A. Cafeína: Revisão sobre Métodos de análises. Química Nova, v.30, n.01, p.99-105. 2007.

POSSER, Y. M. Degradação da cafeína através de processos oxidativos avançados feton e foto feton. Dissertação (Mestre em Engenharia Química), Escola de Engenharia, Universidade Federal do Rio Grande de Sul. Porto Alegre, 2016.

REIS, R. C., DEVILLA, I. A., CORRÊA, P. C., ASCHEU, D. P. R., SÉRVUlO, A. C. D. Propriedades mecânicas de filmes biodegradáveis de fécula de inhame e glicerol. In: Anais de IX Seminário de Iniciação Cientifica, VI Jornada de Pesquisa e Pós-Graduação e Semana Nacional de

$\begin{array}{lrr}\text { ENEPE UFGD e } 5^{\circ} \text { EPEX UEMS (ENCONTRO DE } \\ \text { ENSINO, PESQUISA } & \text { E } & \text { EXTENSÃO } \\ \text { UNIVERSITÁRIA). } & 2014 . & \text { Disponível }\end{array}$ em: $<$ http://eventos.ufgd.edu.br/

enepex/anais/arquivos/150.pdf $>$. Acesso em: 15 de jul 2018.

SILVA. M. H. Efeitos da radiação gama do cobalto60 em sementes de café arábica e conillon: avaliação físico-química. Dissertação (Mestrado em Ciências) - Centro de Energia Nuclear na Agricultura, Universidade de São Paulo. Piracicaba, 2012

StatSoft, Inc. STATISTICA - data analysis software system, version 10. www.statsoft.com. E.U.A. 2011. SUEIRO, A. C. et al. Filmes Biodegradáveis de amido de mandioca, pululano e celulose bacteriana. Química Nova. v.39, n.9, p.1059-2064. 2016.

VERSICO, F. GARCIA, M. A. Cassava (Manihol esculenta) starch films reinforced with natural fibrous filler. Industrial Crops and Products, v.58, p.305314, 2014.
Ciência e Tecnologia. Universidade Estadual de Goiás. 2011.

RIBEIRO. W. X. Caracterização de biofilme à base de zeína e ácido oleico adicionado de nanocarbonato. Dissertação (Mestre e Engenharia e Ciência de Alimentos), Instituto de Biociência, Letras e Ciências Exatas da Universidade Estadual Paulista. São José do Rio Preto. 2014.

SANTOS, P. V. Elaboração, ativação e caracterização de biofilmes à base de fécula de mandioca. Tese (Doutor em Ciência de Alimentos) Faculdade de Engenharia de Alimentos, Universidade Estadual de Campinas, Campinas. 2004.

SANTOS, A. F. Produção de filmes ativos a base de amido e zeólito modificado com prata. Disponível em:http://repositorio.roca.utfpr.edu.br/jspui/bitstream/ 1/3571/1/CM_COEAL_1_01.pdf. Acesso em: 26 jul 2018

SILVA, A. L. Ferreira; ROZA, C. R; Uso da Irradiação em Alimentos: Revisão Boletim do Centro de Pesquisa de Processamento de Alimentos, Curitiba, v.28, n.1, p.49-56, jan.ljun. 2010.

SILVA, G. F. A. CORTEZ-VERGA, W. R. Avaliação da permeabilidade ao vapor de água de filmes nanocompósitos de fécula de mandioca incorporado com óleo essencial de cravo-da-índia (Syzygium aromaticum). In: ENEPEX 2014 - $8^{\circ}$

VICENTINI, M. M. Elaboração e caracterização de filmes comestível à base de fécula de mandioca para uso em póscolheita. Tese (Doutor em Agronomia) Faculdade de Ciências Agronômicas, Universidade Estadual de São Paulo. Botucatu, 2003 